$\underline{\mathbf{P}-130}$

\title{
Infection Induced Alteration in Oil Palm Leaf Metabolites
}

\author{
Nalisha Ithnin ${ }^{1,2,}{ }^{*}$, Intan Safinar Ismail ${ }^{1}$ and Hirzun Md Yusof ${ }^{2, *}$ \\ ${ }^{1}$ Laboratory of Natural Products, Institute of Biosciences, Universiti Putra Malaysia, Serdang, Selangor; ${ }^{2}$ Sime Darby \\ Technology Centre Sdn Bhd, Block B, UPM-MTDC Technology Center III, Universiti Putra Malaysia, Serdang, \\ Selangor; E-mails: intan@science.upm.edu.my,hirzun.mohd.yusof@simedarby.com
}

A metabolite profiling approach based on ${ }^{1} \mathrm{H}$ NMR was used to investigate time-dependent metabolic changes during Ganoderma boninense $(\mathrm{Gb})$ infection in oil palm leaf samples. Leaf samples taken in every month starting from 3 month old seedlings were flashed frozen in liquid nitrogen at sampling site and were later subjected to grinding, extraction in $\mathrm{CD}_{3} \mathrm{OD}+$ $0.05 \%(\mathrm{v} / \mathrm{v})$ TMS and filtration procedures to obtain less polar metabolites. Using biostatistical analysis, differences between non-Gb inoculated and $\mathrm{Gb}$ inoculated samples were found to be insignificant, similarly with the phenotypic observation for both. However, disease progression analysis indicated the up-regulation of amino acid compounds such as 2-aminobutyrate, carnitine, glutamate and $O$-acetylcarnitine and down regulation of sugar and some organic acid compounds throughout the 5 months post infection (mpi). Further understanding of each compound related to plant disease signaling is under investigation.

Keywords: Oil palm, Ganoderma boninense, ${ }^{1} \mathrm{H}$ NMR, Amino acids, Sugars. 